\title{
Light Absorption and Photoluminescence in Quantum Dots and Artificial Molecules
}

\author{
D.A. Firsov ${ }^{a, *}$, L.E. VorobJev ${ }^{a}$, V.Yu. Panevin ${ }^{a}$, \\ N.K. Fedosov ${ }^{a}$, V.A. Shalygin ${ }^{a}$, Yu.B. Samsonenko ${ }^{b}$, \\ A.A. Tonkikh ${ }^{b}$, G.E. Cirlin $^{b}$, A. Andreev ${ }^{c}$, \\ N.V. Kryzhanovskaya ${ }^{c}$, I.S. Tarasov ${ }^{c}$, N.A. Pikhtin ${ }^{c}$, \\ V.M. Ustinov ${ }^{c}$, S. Hanna ${ }^{d}$, A. Seilmeier ${ }^{d}$, F.H. Julien ${ }^{e}$, \\ N.D. ZAKHAROV ${ }^{f}$ AND P. WERNER ${ }^{f}$ \\ ${ }^{a}$ St. Petersburg State Polytechnic University, 195251 St. Petersburg, Russia \\ ${ }^{b}$ Institute for Analytical Instrumentation RAS, 198103 St. Petersburg, Russia \\ ${ }^{c}$ Ioffe Physico-Technical Institute, RAS, 194021 St. Petersburg, Russia \\ ${ }^{d}$ Institute of Physics, University of Bayreuth, 95440 Bayreuth, Germany \\ ${ }^{e}$ Institut d'Eléctronique Fondamentale, Université Paris-Sud, 91405 Orsay, France \\ ${ }^{f}$ Max Planck Institute of Microstructure Physics, 06120 Halle (Saale), Germany \\ Intraband absorption in $n$ - and $p$-doped structures with InAs/GaAs \\ quantum dots covered by InGaAs layers is studied both experimentally and \\ theoretically. The absorption cross-section for $p$-type quantum dots was \\ found to be significantly smaller than that for $n$-type quantum dots. Inter- \\ band absorption bleaching under strong interband excitation is found and \\ investigated in undoped quantum dot structures. Structures with artificial \\ molecules were grown. Photoluminescence spectra and transmission elec- \\ tron microscopy images proves the presence of coupled symmetrical quantum \\ dots. \\ PACS numbers: 78.30.Fs
}

\section{Introduction}

Quantum dot (QD) mid-infrared devices based on intraband electron transitions can have parameters better than quantum well detectors and cascade lasers because of a long electron lifetime in excited localized and extended states of QD. There are no experimental or theoretical comparative studies of electron and hole intraband light absorption in InAs/GaAs QDs. Meanwhile, such studies may be

*corresponding author; e-mail: dmfir@rphf.spbstu.ru 
useful for the development of mid-infrared QD devices and evaluation of optical losses related to hole absorption in injection mid-infrared QD lasers based on intraband interlevel optical electron transitions in QDs $[1,2]$. In the present work the results of experimental and theoretical studies of intraband absorption in $n$ and $p$-doped InAs/GaAs QDs covered by InGaAs quantum wells are presented. Bleaching the interband absorption under powerful interband optical pumping was also observed in QDs.

The works devoted to the quantum dot molecules (QDMs) are few in number in spite of the fact that QDMs can serve as a basis for new mid-infrared and terahertz emitters because the energy level splitting in QDM is a function of spacer thickness and lies in the range from tens $m e V$ to a few meV. We present some preliminary results on the growth and investigations of InAs/GaAs QDM structures.

\section{Samples and techniques}

The structures containing 15 QD layers grown on GaAs (001) substrate by MBE were investigated. In contrast to conventional Stranski-Krastanov growth, QDs were overgrown with $5 \mathrm{~nm}$ thick $\mathrm{In}_{0.12} \mathrm{Ga}_{0.88}$ As layer. This procedure modifies structural and optical properties of InAs QDs. As it was shown in [3], QDs grown by this technique represent truncated pyramids of enlarged volume. Doped structures contain $2 \mathrm{~nm}$ thick Be or Si doped layers for $p$ - and $n$-types of QDs, located $1.5 \mathrm{~nm}$ below the QD layers. The sheet doping level, $n_{\mathrm{Be} \text { (or Si) }}=1.8 \times 10^{11} \mathrm{~cm}^{-2}$, exceeds the dot concentration by a few times. The light absorption was studied for $s$ - and $p$-light polarization in multipass geometry.

In order to form QDMs, two layers of InAs/GaAs QDs separated by a $5 \mathrm{~nm}$ spacer were grown. The amount of deposited material in the first and second QD layers was different in order to provide the shape and dimension uniformity of QDs in both layers. Photoluminescence spectra and transmission electron microscopy (TEM) images were studied in QDM structure.

\section{Intraband light absorption in QD structures}

Spectral dependences of the ratio of the transparences for $p$ - and $s$-polarized light are presented in Fig. 1 for $p$-, $n$-, and undoped samples. One can see a clear minimum and maximum in the spectrum of $T_{p} / T_{s}$ for $n$-doped sample corresponding to dominating absorption of $p$ - and $s$-light polarization, respectively (shown by arrows). No significant features are presented in the two other spectra, so we can conclude that the hole related absorption is considerably less than electron one.

Calculations of carrier energy spectra, wave functions, and optical matrix elements were made in a frame of the envelope wave function method based on $8 \times 8$ effective Kane Hamiltonian taking into account strain and piezoelectric fields. The QD shape was assumed to be a square truncated pyramid with the base of $16 \times 16 \mathrm{~nm}^{2}$, the top of $4 \times 4 \mathrm{~nm}^{2}$ and the height of $5.5 \mathrm{~nm}$. Optical matrix 


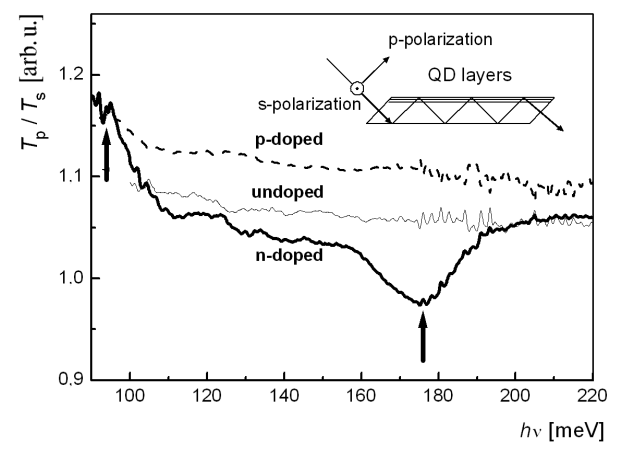

Fig. 1. Spectral dependences of the ratio of light transmissions for $p$ - and $s$-polarizations $T_{p} / T_{s}$ in QD samples with different doping types at $T=300 \mathrm{~K}$. The main electron transitions are shown with arrows. Diagram of the experiment is shown in the inset.
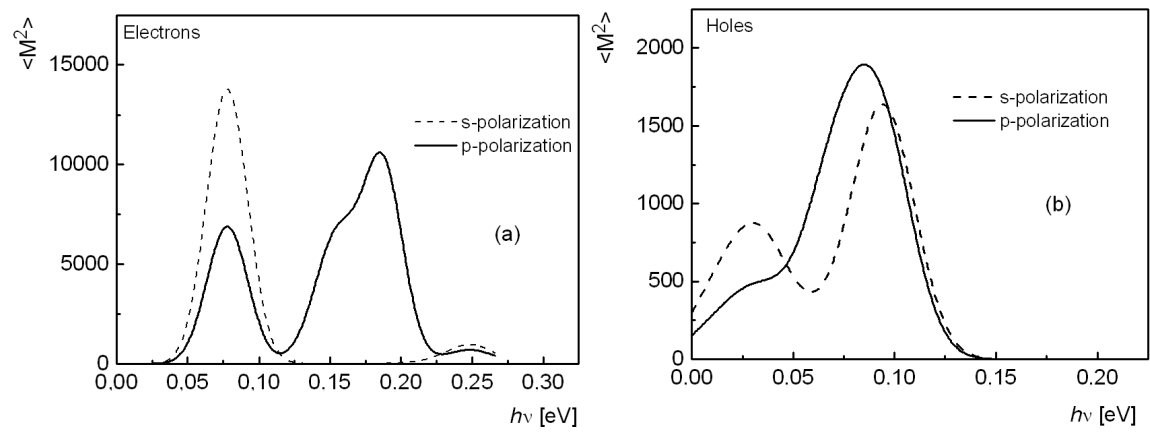

Fig. 2. Averaged squared matrix elements for electron (a) and hole (b) transitions in QDs for $p$ - and $s$-polarized light.

elements were calculated for carrier intraband transitions under radiation with $x, y$, and $z$-polarizations ( $z$ axis is parallel to the structure growth direction, $x$ and $y$ axes lie in the plane of the structure). In order to compare experimental and theoretical results these data were recalculated for $s$-and $p$-polarized light and broadened with a broadening factor of about $20 \mathrm{meV}$. The results are presented in Fig. 2. As it follows from calculations for electrons, the absorption peak for $s$-polarized light is connected with electron transitions between ground $|000\rangle$ and excited $|100\rangle$ and $|010\rangle$ states, and the absorption peak for $p$-polarized light is connected with transitions $|000\rangle \rightarrow|001\rangle$ allowed for $z$ polarized light.

We can conclude that the light absorption connected with hole transitions is significantly smaller in comparison with that connected with electron transitions. Besides, the light absorption for electron and hole transitions has different polarization and spectral dependences. At $h \nu=180 \mathrm{meV}$ absorption cross-section per one electron in QD corresponding to electron transitions $|000\rangle \rightarrow|001\rangle$ is equal to $4.1 \times 10^{-15} \mathrm{~cm}^{2}$. 


\section{Photoinduced modulation of interband light absorption in QDs}

The spectrum of equilibrium interband light absorption was measured in undoped QD structure for unpolarized light (see Fig. 3a). It has pronounced peaks connected with transitions between ground as well as first excited states in valence and conduction bands. Under intensive generation of $\mathrm{e}-\mathrm{h}$ pairs with interband laser pumping the ground electron and heavy hole states are filled. This leads to bleaching the sample and light amplification if the probe light beam is tuned on spectral region of the first absorption peak. The decrease of the absorption is connected with Pauli blocking the interband interlevel electron transitions.
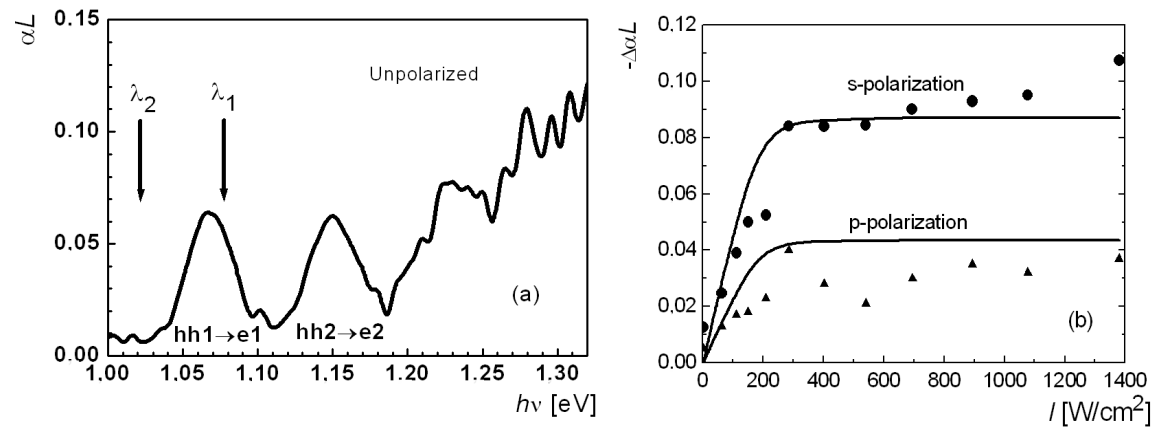

Fig. 3. Equilibrium interband absorption spectrum of undoped QD structure ( $\alpha$ is absorption coefficient, $L$ is optical path), $\lambda_{1}$ and $\lambda_{2}$ are wavelengths of probing lasers (a); change of absorbance at wavelength $\lambda_{1}$ for $s$ - and $p$-polarized light versus intensity of interband optical pumping (b) (solid lines are shown for eyes). $T=77 \mathrm{~K}$.

We used two semiconductor lasers for probing with wavelengths $\lambda_{1}=$ $1.15 \mu \mathrm{m}$ and $\lambda_{2}=1.215 \mu \mathrm{m}$ belonging to the spectral range of the absorption peak $h h 1 \rightarrow e 1$ and outside of them, respectively (see Fig. 3a). The change of absorbance $\Delta \alpha L$ for light with wavelength $\lambda_{1}$ as a function of optical pumping intensity is shown in Fig. 3b. The change of absorption of $p$-polarized light in saturation region is two times less than for $s$-polarized light because optical transitions for light polarized along growth axis $z$ are forbidden. The absorption of probe light at $\lambda_{2}$ is caused by nonequilibrium carriers outside of QDs and weakly changes under interband pumping.

\section{Artificial QD molecules}

We present preliminary results on growing and characterizing the structures with QD molecules. Energy splitting of the ground level of QDs forming an artificial molecule is a function of spacer thickness. As it follows from our calculations, for the $5 \mathrm{~nm}$ spacer this splitting is around $45 \mathrm{meV}$. The photoluminescence spectra of QDM structure measured over a wide interval of pumping intensity are shown in Fig. 4. The behavior of the spectra at weak excitation proves the formation of 


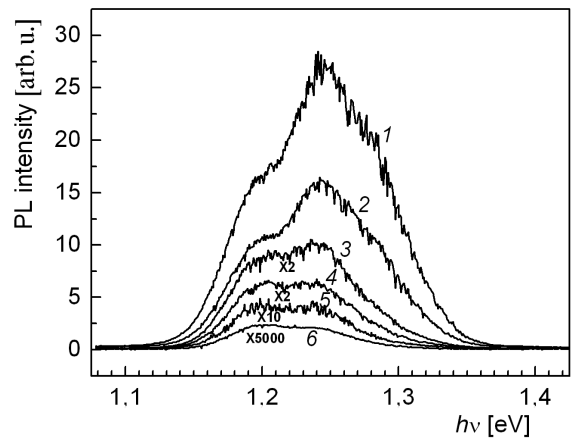

Fig. 4. PL spectra measured in QDM structure at different excitation levels: $1-I_{0}$, $2-I_{0} / 2,3-I_{0} / 7,4-I_{0} / 50,5-I_{0} / 500,6-I_{0} / 250000 . T=77 \mathrm{~K}$.

the molecule and the presence of a split state with an energy splitting of $42 \mathrm{meV}$. High resolution TEM images also validate the presence of coupled symmetrical QDs in our structure.

\section{Conclusion}

In summary, the results shown above prove a weak intraband absorption in QD structures connected with hole transitions in comparison with electron ones. Interband absorption bleaching under powerful interband excitation allows one to find the pumping intensity corresponding to filling QD ground states. Artificial QD molecules suitable for the development of $\mathrm{THz}$ devices can be grown using an appropriate technology.

\section{Acknowledgment}

This work was supported by RFBR, Russian Ministry of Education and Science and INTAS. The work of A. Andreev was partially supported by EPSRC $(\mathrm{UK})$.

\section{References}

[1] L.E. Vorobjev, JETP Lett. 68, 417 (1998).

[2] A. Kastalsky, L.E. Vorobjev, D.A. Firsov, V.L. Zerova, E. Towe, IEEE J. Quantum Electron. 37, 1356 (2001).

[3] M.V. Maximov, A.F. Tsatsul'nikov, B.V. Volovik, D.S. Sizov, Yu.M. Shernyakov, I.N. Kaiander, A.E. Zhukov, A.R. Kovsh, S.S. Mikhrin, V.M. Ustinov, Zh.I. Alferov, Phys. Rev. B 62, 16671 (2000). 